Technical note

\title{
A structural analytic method on the phase space data of Linac 4 MV photons based on the real world
}

\author{
Zhenguo Cui, Songlin Sha, Yanling Bai * \\ Department of Radiation Physics, Harbin Medical University Cancer Hospital, 150081 Harbin, Heilongjiang Province, PR China
}

\section{A R T I C L E I N F O}

\section{Keywords:}

Monte Carlo

Linac

Phase Space $(\mathrm{PhSp})$

Geant4

\begin{abstract}
A B S T R A C T
Purpose: It was given that the characteristics of the fluence distribution and the energy spectrum structure of 4MV photons on the Phase Space (PhSp) plane and a method to analyzing the characteristics.

Methods: After the PhSp file of 4 MV photons was acquired by the method of Monte Carlo (MC) calculation, the photons recorded by $\mathrm{PhSp}$ file were grouped based on the energy bin, and it was analyzed that the spatial distribution and energy spectrum structure of the photons. The photons in each energy group were continually grouped to sub-files according to momentum bin, and the primary and scattered photons could be separated according to the character of the fluence distribution of the photons in the sub-files.

Results: The energy of 4 MV beam is a continuous spectrum. The energy constituent on a pixel at different distances from the center point is different, and the average energy on the center axis of the field is the highest; The photons with $0-1.0 \mathrm{MeV}$ had $42.6 \%$ of all; that with energy more than $3.0 \mathrm{MeV}$ had $11.7 \%$; greater than $4 \mathrm{MeV}$, just $1.5 \%$. The primary and scattered photons were easy collected according to the distribution characteristics of sub-groups.

Conclusions: The work to acquire and analyze the PhSp file of the $4 \mathrm{MV}$ beam is significant. $4 \mathrm{MV}, 6 \mathrm{MV}, 8 \mathrm{MV}, 10$ MV and 15 MV energy beams basically cover the beams of radiotherapy, and a database of the energy beams could be built for the MC related research of other scholars.
\end{abstract}

\section{Introduction}

Medical linear accelerator (Linac) is one of the most crucial radiation source devices in radiation therapy [1]. The research of the radiation dosimetry of Linac has become essential projects in medical physics field. Many algorithms of radiation dose calculation have been applied clinically at present [2-4]. However, they are lack of the accuracy in heterogeneous medium when compared with the Monte Carlo (MC) method [5].

Although MC method has the advantage in precise, the process of the dose calculation by this method is often time-consuming. The computation efficiency could be improved by the application of the Phase Space (PhSp) file as the input source in the MC simulation [6]. The PhSp file records the information of the particle state (type, position coordinate, momentum direction, energy, etc) $[7,8]$. A vast photon is needed to ensure a fine variance of the photon fluence distribution, which requires a large storage space and takes a long time to load the $\mathrm{PhSp}$ data into the memory from hard disk in the dose calculation. The disadvantage of the using PhSp file could be avoided using the parameter model of source in the dose calculation $[9,10]$.

There was a lack of studies about the PhSp files of 4 MV beam of Linac. The purpose of this study was to present a method to analyze the characteristics of the spatial distribution and the energy spectrum of 4 MV beam. This study was only a prelude to the follow-up research which included the construction of a unique virtual source model, the optimization of $\mathrm{PhSp}$ variance, and the influence of $\mathrm{PhSp}$ variance transferred on the expected convergence rate of MC dose calculation.

\section{Materials and methods}

\subsection{The platform and the physics parameters in $M C$ simulation}

This research project was performed on a DELL T640 Server computer with a 196-GB memory, double 24-core CPUs with 96 threads, and Ubuntu 19.4 operation system. MC simulations were performed using Geant4.10.0 [11]. The simulated physical process included the Electromagnetic (EM) interaction and the hadronic interaction. The EM model consisted of Compton scatter, photoelectric effect, gamma

\footnotetext{
* Corresponding author: Department of Radiation Physics, Harbin Medical University Cancer Hospital, Harbin, China.

E-mail address: baiyl2126@126.com (Y. Bai).
} 
conversion, and Rayleigh scatter. The production threshold was set to 1 $\mathrm{mm}$ for electrons, positrons and photons. The spatial distribution of the electron current was in the form of Gaussian, and the peak full width at half maximum (FWHM) was $1.2 \mathrm{~mm}$ for $4 \mathrm{MV}$ beam; its energy distribution was also in the form of Gaussian function, most probable energy was $4.5 \mathrm{MeV}$, and the FWHM of energy was $0.2123 \mathrm{MeV}$. A total of $5.4 \times 10^{10}$ electrons were simulated, and $1.3 \times 10^{9}$ photons had been recorded in the $\mathrm{PhSp}$ file. The photon information parameters included the spatial coordinates $(\mathrm{x}, \mathrm{y}, \mathrm{z})$, energy $(\mathrm{E})$, and momentum orientation $\left(\widehat{P}_{x}, \widehat{P}_{y}, \widehat{P}_{z}\right)$ of the photons across the PhSp plane. The process of acquiring the $\mathrm{PhSp}$ file took about $10 \mathrm{~h}$. A small number of secondary electrons were generated during the interaction between the photons and the internal components of the accelerator head. Although they went across the $\mathrm{PhSp}$ plane, they had a negligible impact on the surface dose of the water phantom and had been ignored in the $\mathrm{PhSp}$ file.

\subsection{Accelerator treatment head geometric model}

The geometric parameters of the treatment heads components were provided by the Elekta Company in Sweden and were authorized to be used in our relevant research. The Linac target was made of an alloy that contained $90 \%$ of tungsten, and it had a diameter of $5.4 \mathrm{~mm}$ and a thickness of $0.89 \mathrm{~mm}$. The primary collimator was a cylindrical tungsten alloy with a thickness of $101.1 \mathrm{~mm}$ and an outer diameter of $190.0 \mathrm{~mm}$. It had a cone hole with the upper diameter of $12.9 \mathrm{~mm}$ and the lower diameter of $62.9 \mathrm{~mm}$, whose function was to determine the projection range of the Bremsstrahlung photons from the target. The flattening filter was made of stainless steel $(99.3 \% \mathrm{Fe})$ and was used to generate a flat lateral dose profile. It was a conic shape solid with a height of $17 \mathrm{~mm}$ and a basal diameter of $65 \mathrm{~mm}$. The secondary collimators consisted of MLCs (The Multi-Leaf Collimator system) and two pairs of bank jaws located beneath the MLCs, the projection width of a leaf was $10 \mathrm{~mm}$ on the isocenter plane. MLCs bank jaws were also made of tungsten alloy with thicknesses of $75 \mathrm{~mm}$ and $78 \mathrm{~mm}$, respectively.

The PhSp plane, with an area of $100 \times 100 \mathrm{~mm}^{2}$, was placed between the flatten filter and MLCs, and was $175 \mathrm{~mm}$ from the bottom of the Tungsten target. To ensure the universality of the PhSp file for other Linacs, the MLCs and bank jaws were removed away when obtaining the PhSp file. The PhSp file could be used by the combination with the treatment head model of other Linac from different manufactures in MC dose calculation because it did not include the influencing factors from the secondary collimator.

\subsection{To split PhSp data based on photon energy}

The energy of the Linac 4 MV X-ray was a continuous spectrum from 0 to $5 \mathrm{MeV}$. The photons on the PhSp plane were divided into 20 energy groups $\mathrm{PhSp}$ sub-files by the energy interval $\operatorname{Bin}_{E}=0.25 \mathrm{MeV}$. The photon energy range in the ith energy group was $\left[E_{\min }, E_{\max }\right] . E_{\min }$ and $E_{\max }$ respectively represented the minimum and maximum energy of the photons, and they could be written as the following functions:

$E_{\text {min }}=(i-1) \times \operatorname{Bin}_{E}(i=1,2, \ldots, 20)$

$E_{\max }=i \times \operatorname{Bin}_{E}(i=1,2, \ldots, 20)$

\subsection{To split PhSp data based on the photon momentum}

$\left(\widehat{P}_{x}, \widehat{P}_{y}, \widehat{P}_{z}\right)$ were three projection values of unit momentum of a photon on the $\mathrm{x}, \mathrm{y}, \mathrm{z}$ coordinate axis. The photons acquired on $\mathrm{PhSp}$ plane were grouped by the interval $\operatorname{Bin}_{\widehat{P}_{z}}$ of momentum projection $\widehat{P}_{z}$, and the listing of $\operatorname{Bin}_{\widehat{P}_{z}}$ were shown in the function (3).
$\operatorname{Bin}_{\widehat{P}_{z}}=\left\{\begin{array}{l}0.001\left(0.998 \leq \widehat{P}_{z}<1.000\right) \\ 0.002\left(0.992 \leq \widehat{P}_{z}<0.998\right) \\ 0.004\left(0.960 \leq \widehat{P}_{z}<0.992\right) \\ 0.020\left(0.920 \leq \widehat{P}_{z}<0.960\right)\end{array}\right.$

Every energy group was divided into 17 momentum groups $\mathrm{PhSp}$. Very tiny amounts of photons with $\widehat{P}_{z}<0.920$ were projected beyond the maximum irradiation field size on the isocenter plane. Hence, these photons had been rejected from the PhSp file. The total reference PhSp data were divided into $340 \mathrm{PhSp}$ sub-files finally.

\section{Results}

\subsection{The results of segmented PhSp data by the energy $\operatorname{Bin}_{E}$}

The distribution form of photons was analyzed by probability density. The probability density of photons was defined as the ratio of the number of photons in a pixel to the total number of photons in a PhSp plane, and it could be denoted as:

$p\left(x, y, E_{i}\right)=N_{\text {pixel }}\left(x, y, E_{i}\right) / N_{0}$

where $N_{\text {pixel }}$ and $N_{0}$ represented the number of photons in a certain pixel and the total number of photons in the PhSp plane. The photon counts in the ith energy group could be described as:

$N_{i}\left(E_{i}\right)=N_{0} \iint p_{i}\left(x, y, E_{i}\right) d x d y$

Then the total number of photons in the $\mathrm{PhSp}$ plane was expressed by the following function.

$N_{0}=\sum_{i=1}^{i=20} N_{i}\left(E_{i}\right)$

The cross-sectional views of the photon distribution of every energy group on the PhSp plane were shown in Fig. 1. The photons count of per group of twenty energy groups $\mathrm{PhSp}$ were normalized to the photons counts on the center pixel of the second energy group.

As was shown in Fig. 1. The line A and line B represented two position points (or pixels) on the PhSp plane, respectively. After line A and $B$ met with the profile curves of the photons fluence of the 20 energy groups PhSp, there were twenty intersections. The ratio relationship of photons fluence at the twenty intersections point constituted the energy spectrum for A and B pixel spots. It was known that the energy spectra on the different pixel was the function of the distances from the center of the irradiation field.

\subsection{The results of segmented PhSp data by the momentum Bin ${\widehat{P_{z}}}$}

Based on the methods described in the second section, every energy group was divided into the 17 sub-momentum groups $\mathrm{PhSp}$ by the moment $\operatorname{Bin}_{\widehat{P}_{z}}$. The photon distribution of four special momentum groups PhSp were shown in Fig. 2.

As Fig. 2 showed, most photons in each PhSp sub-file distributed in the annular region and they were regarded as the primary photons. The emission point of them focused on tungsten targets. However, there were a tiny number of photons which randomly distributed across the entire $\mathrm{PhSp}$ plane, and their emission points could not focus on the tungsten target. They were regarded as the scattered photons which were produced by the interaction of photons with flatten filters and primary collimator. The primary photons count of the jth momentum group in the ith energy group was expressed as the following function:

$N_{\text {primary }}\left(E_{i}, P_{z, j}\right)=N_{0} \int_{r_{1}}^{r_{2}} \int_{0}^{2 \pi} p_{i j}\left(E_{i}, P_{z, j}, r, \theta\right) d r d \theta$ 


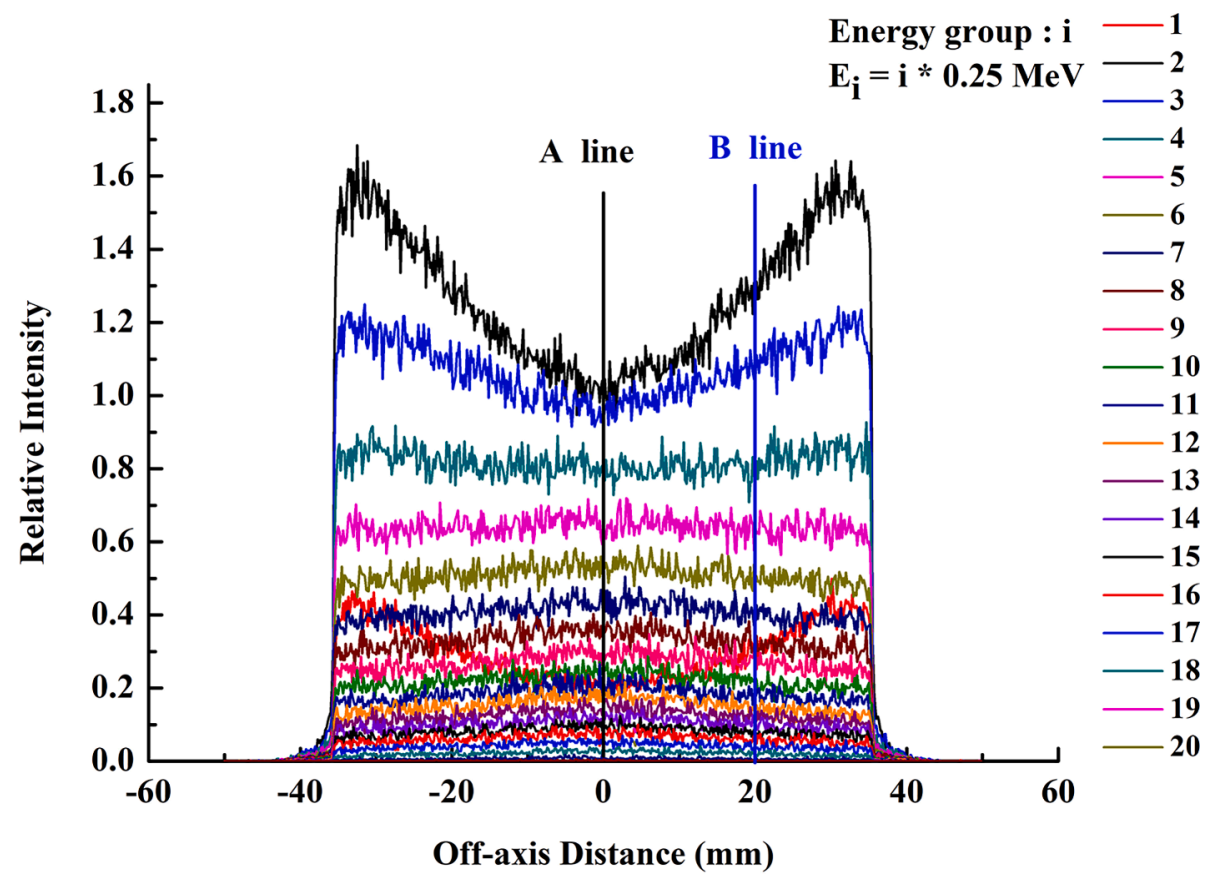

Fig. 1. The profile of the spatial distribution of photons in twenty energy groups $\mathrm{PhSp}$.
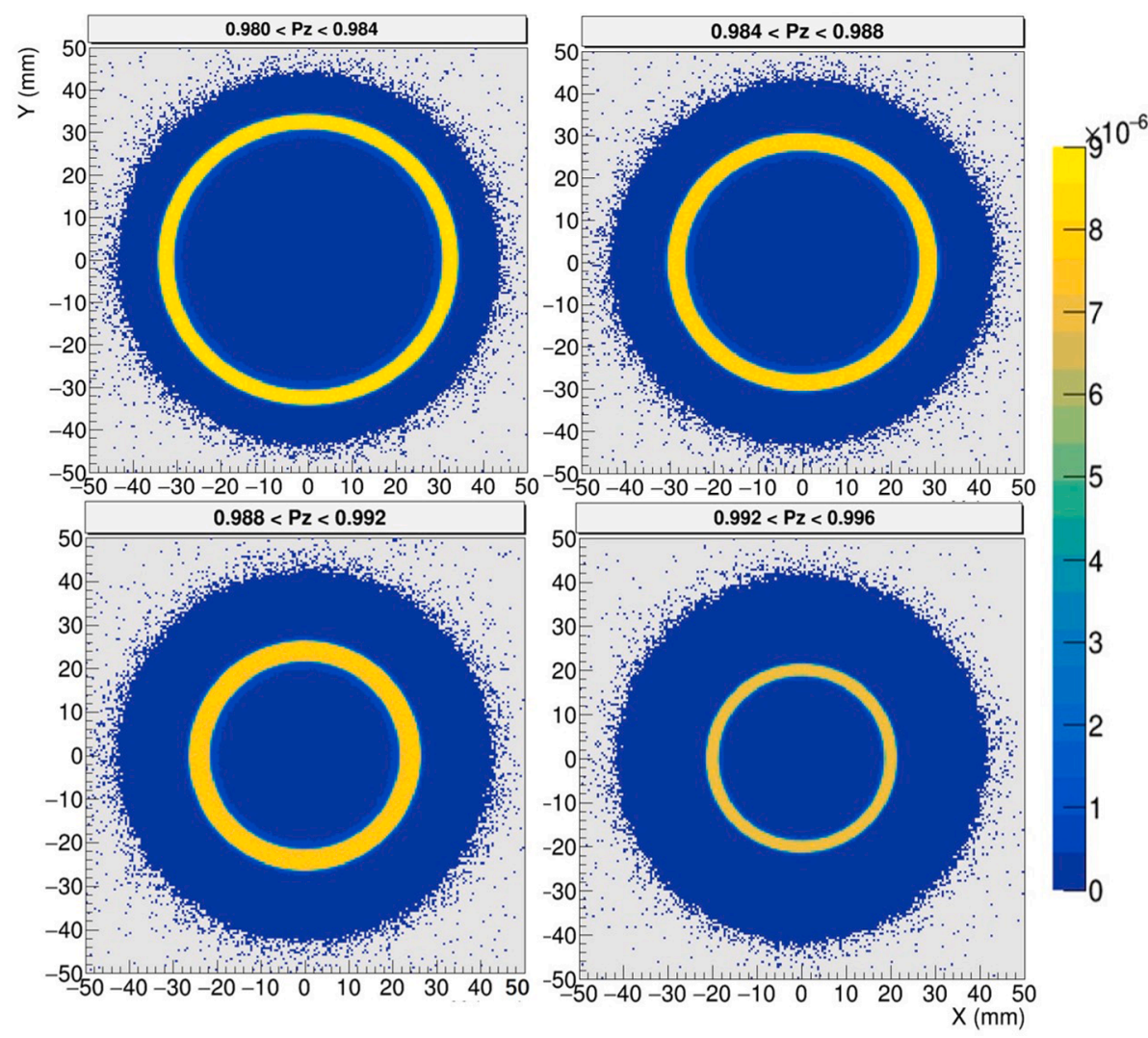

Fig. 2. Photon probability density distributions in the 4 different momentum groups PhSp from the third energy group with the $0.5-0.75$ MeV of photons.

where $r_{1}$ and $r_{2}$ stood for the inner and outer boundary of the annular highlight region, respectively. $p_{i j}\left(E_{i}, P_{z, j}, r, \theta\right)$ represented the probability density of the jth momentum group in the ith energy group. Because the photon distribution in each PhSp sub-file presented spatial symmetry, the number of primary photons could be expressed by the following function:
$N_{\text {primary }}\left(E_{i}, P_{z, j}\right)=2 \pi N_{0} \int_{r_{1}}^{r_{2}} p_{i j}\left(E_{i}, P_{z, j}, r\right) d r$

Hence, the number of the scatter photons could be acquired by the following function: 
$N_{\text {scatter }}\left(E_{i}, P_{z, j}\right)=2 \pi N_{0} \int_{0}^{r} p_{i j}\left(E_{i}, P_{z, j}, r\right) d r-N_{\text {primary }}\left(E_{i}, P_{z, j}\right)$

\subsection{Validation of the PhSp data}

All measurements were implemented by the IBA II Blue Phantom with a dimension of $480 \times 480 \times 410 \mathrm{~mm}^{3}$ utilizing continuous data acquisition mode. An ionization chamber cc13 (IBA) with active volume $0.13 \mathrm{~cm}^{3}$ was selected, and SSD $=\mathrm{SAD}=1000 \mathrm{~mm}$. The size of two beam fields on the isocenter plane of the accelerator was $300 \times$ $300 \mathrm{~mm}^{2}$ and $100 \times 100 \mathrm{~mm}^{2}$, respectively.

The data of the measurement and simulation involved the percent depth dose (PDDs) and lateral dose profiles at the four different depths, and the results were normalized to the maximum dose on the central axis of each irradiation field. The number of the valid photons was only $7.3 \times 10^{7}$ for $100 \times 100 \mathrm{~mm}^{2}$ beam field; it was $5.5 \times 10^{8}$ for $300 \times$ $300 \mathrm{~mm}^{2}$ beam field. The statistical fluctuations of the photon count in the $\mathrm{PhSp}$ were regarded as 'latent variance' because of the fixed number of photons in a PhSp file, and it was one of the reasons which led to the fluctuation of the dose distribution in the phantom [12]. The relevant discussion had been done in a previous study [13]. The variance of the dose calculation in the MC simulation was inversely proportional to the fluence density of the particle incident in a phantom. In order to reduce the variance of the dose calculation, photons were recycling 35 times. The random seed calculated in each cycle was different so that each recycling result was independent. The size of the cubic water phantom was $400 \times 400 \times 400 \mathrm{~mm}^{3}$, and the size of every voxel was $2 \times 2 \times$ $2 \mathrm{~mm}^{3}$. The calculation was performed in the case of the $100 \times$ $100 \mathrm{~mm}^{2}$ and $300 \times 300 \mathrm{~mm}^{2}$ beam fields. PDDs were showed in Fig. 3, and the worse agreement of the dose was locally observed on the water surface. The maximum discrepancies of the difference between the calculation and measurement were as high as $6 \%$ and $5 \%$ in the case of two beam fields, respectively. The reason was that the measurement result on the surface of the water phantom was incorrect due to the volume effect of the ion chamber and secondary electronic nonequilibrium factors in the surface region of the water phantom. The percentage differences did not exceed $2.0 \%$ except the surface region; moreover, it gradually decreased as the depth increased.

Fig. 4 showed the comparison of the lateral dose profile between the measurement and the simulation at the four different depths $(1.0 \mathrm{~cm}, 5$ $\mathrm{cm}, 10 \mathrm{~cm}$, and $20 \mathrm{~cm}$ ) respectively. The results were normalized to the maximum dose at the depth $1.0 \mathrm{~cm}$ on the central axis. In the range of the $80 \%$ of the dose of the central axis, the maximum discrepancy of the
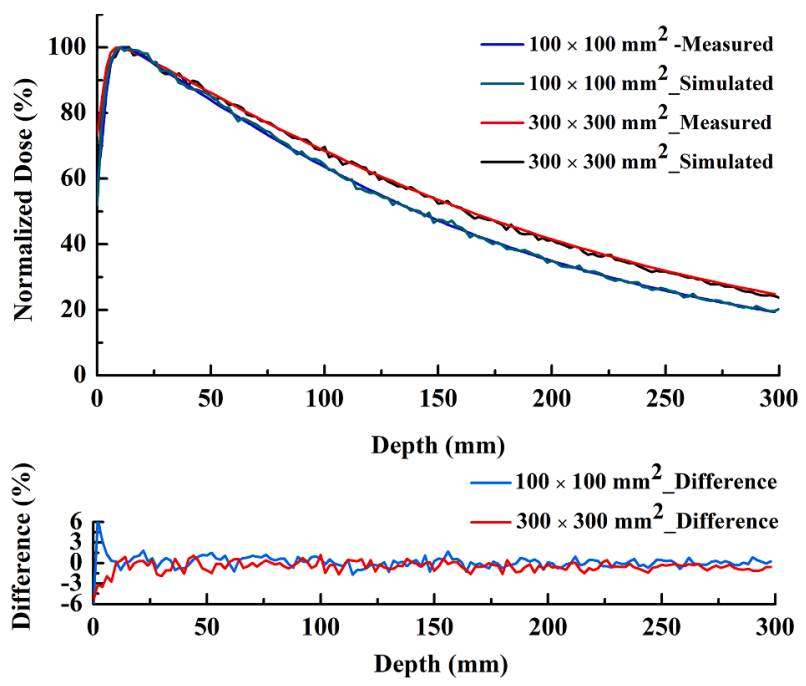

Fig. 3. Comparison of the PDD and the difference between the simulated and measurement.

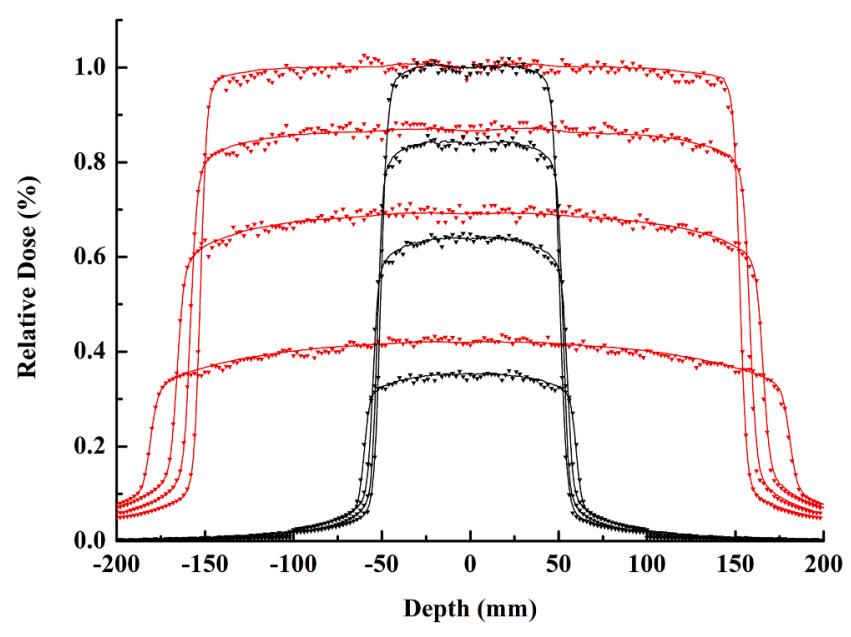

Fig. 4. Comparison of the dose profile along the $\mathrm{X}$-axis between calculation and measurement at the four different depths in water. The red and the black represented the $300 \times 300 \mathrm{~mm}^{2}$ and the $100 \times 100 \mathrm{~mm}^{2}$ beam field, respectively. The depth was $1 \mathrm{~cm}, 5 \mathrm{~cm}, 10 \mathrm{~cm}$, and $20 \mathrm{~cm}$ from high to low. (For interpretation of the references to colour in this figure legend, the reader is referred to the web version of this article.)

relative variances was $1.7 \%$ between the measured and the simulated in the case of the field size $100 \times 100 \mathrm{~mm}^{2}$ while it was $1.4 \%$ for the field size $300 \times 300 \mathrm{~mm}^{2}$. The calculated dose presented great agreement with the measured dose. The consistency of the PDD and the lateral dose profile between the measurement and simulated indicated that the data of the PhSp file obtained accurately reflected the characteristic of the photons source of the accelerator from the real-world and could be used as the photon input source in the dose calculation.

\section{Discussions}

The PhSp file has been extensively used to be as the input source in the dose calculation. To obtain the PhSp file by the MC simulation, the relevant geometric information inside the treatment head of the Linac must be known. Due to lack of the geometric parameter information, the $\mathrm{PhSp}$ file for the special type of Linac could be acquired by the optimization of PhSp file which had been gained [14].

There was truly little literature about the PhSp file of 4 MV x-ray until now. As was shown in Fig. 1, for $4 \mathrm{MV}$ x-ray beam, the lower energy photons with the energy range from $0.25 \mathrm{MeV}-1.0 \mathrm{MeV}$ accounted for about $42.6 \%$ of the total photons in the PhSp file; the proportion of photons with energy greater than $3 \mathrm{MeV}$ was little, only $11.7 \%$; and photons with the energy greater than $4 \mathrm{MeV}$ was much less, only $1.5 \%$. Furthermore, the farther away from the center point in $\mathrm{PhSp}$ plane, the greater the proportion of low-energy photons and the lower the average energy. The characteristic was also corroborated by our measuring work of lateral dose profile at different depths. Moreover, it was convenient to analyze the energy spectrum of photons and the characterize of photons fluence distributions on the $\mathrm{PhSp}$ plane by grouping the $\mathrm{PhSp}$ data according to the energy and momentum of the photons, it was also convenient to distinguish the primary photons and the scattered photons based on their emitting position (Fig. 2). This work was also to prepare for the following-up construction of a novel source model, and the use of $\mathrm{PhSp}$ sub-files would be of benefit to the multi-thread process in MC calculation.

For a beam with the same nominal radiation energy produced by any type of Linac, its dose characteristics must meet the relevant requirements of national laws and professional associations. These requirements are only for energy beam, not for Linac models and brands. The secondary collimation systems (MLCs and jaws) of Linac from different manufacturers do not affect the dose specification 
requirements in the irradiation field while it only influences the characteristics of the dose drop off gradient in the boundary area of the irradiation field.

\section{Conclusions}

This study had shown that the discretization of the PhSp data based on the energy and momentum of the photons was a feasible method to analyze the spatial distribution characteristics and the energy spectrum structure of the photons in the PhSp file.

The research on the PhSp file of $4 \mathrm{MV}$ beam was a part of building a database of radiotherapy beams. $4 \mathrm{MV}, 6 \mathrm{MV}, 8 \mathrm{MV}, 10 \mathrm{MV}$ and $15 \mathrm{MV}$ energy beams basically cover the range of the energy beams in radiation therapy field. Hence, a database of the energy beams could be built, which will be greatly helpful for other scholars to use the database in the relevant research of MC calculation.

\section{Acknowledgments}

This research project is funded by the National Natural Science Foundation of China (Grant NO. 11775064) and the China International Medical Foundation (Grant NO. HDRS2020030204); and thank Elekta Company provided the necessary information of Linac models for the work, the research has not any profitable conflicts with other people and organizations.

\section{References}

[1] Sempau J, Acosta E, Baro J, Fernández-Varea JM, Salvat F. An algorithm for Monte Carlo simulation of coupled electron-photon transport. Nucl Instrum Methods Phys Res Sect B Beam Interact with Mater Atoms 1997;132(3):377-90. https://doi.org/ 10.1016/S0168-583X(97)00414-X.

[2] Ahnesjö A, Saxner M, Trepp A. A pencil beam model for photon dose calculation. Med Phys 1992;19(2):263-73. https://doi.org/10.1118/1.596856.
[3] Ulmer W, Pyyry J, Kaissl W. A 3D photon superposition/convolution algorithm and its foundation on results of Monte Carlo calculations. Phys Med Biol 2005;50(8): 1767-90. https://doi.org/10.1088/0031-9155/50/8/010.

[4] Ahnesjö A. Collapsed cone convolution of radiant energy for photon dose calculation in heterogeneous media. Med Phys 1989;16(4):577-92. https://doi, org/10.1118/1.596360.

[5] Chandola RM, Tiwari S, Painuly NK, Choudhary V, Azad SK, Beck M. Monte Carlo study of dosimetric parameters and dose distribution effect of inhomogeneities and source position of GammaMed Plus source. J Cancer Res Ther 2011;7:29-34. https://doi.org/10.4103/0973-1482.80453.

[6] Kim TH, Schaarschmidt T, Yang HJ, Kim YK, Chun KJ, Choi Y, et al. Development of an IAEA phase-space dataset for the Leksell Gamma Knife ${ }^{\circledR}$ Perfexion $^{\mathrm{TM}}$ using multi-threaded Geant4 simulations. Phys Med 2019;64:222-9. https://doi.org/ 10.1016/j.ejmp.2019.07.002.

[7] Ding GX. Energy spectra, angular spread, fluence profiles and dose distributions of 6 and $18 \mathrm{MV}$ photon beams: Results of Monte Carlo simulations for a Varian 2100EX accelerator. Phys Med Biol 2002;47(7):1025-46. https://doi.org/10.1088/ 0031-9155/47/7/303.

[8] Lovelock DMJ, Chui CS, Mohan R. A Monte Carlo model of photon beams used in radiation therapy. Med Phys 1995;22(9):1387-94. https://doi.org/10.1118/ 1.597620 .

[9] Fix MK, Keall PJ, Dawson K, Siebers JV. Monte Carlo source model for photon beam radiotherapy: Photon source characteristics. Med Phys 2004;31(11): 3106-21. https://doi.org/10.1118/1.1803431.

[10] Townson RW, Jia X, Tian Z, Graves YJ, Zavgorodni S, Jiang SB. GPU-based Monte Carlo radiotherapy dose calculation using phase-space sources. Phys Med Biol 2013;58(12):4341-56. https://doi.org/10.1088/0031-9155/58/12/4341.

[11] Cortés-Giraldo MA, Quesada JM, Gallardo MI, Capote R. An implementation to read and write IAEA phase-space files in GEANT4-based simulations. Int J Radiat Biol 2012;88(1-2):200-8. https://doi.org/10.3109/09553002.2011.627977.

[12] Sempau J, Sánchez-Reyes A, Salvat F, Tahar HOB, Jiang SB, Fernández-Varea JM. Monte Carlo simulation of electron beams from an accelerator head using PENELOPE. Phys Med Biol 2001;46(4):1163-86. https://doi.org/10.1088/00319155/46/4/318.

[13] Walters BRB, Kawrakow I, Rogers DWO. History by history statistical estimators in the BEAM code system. Med Phys 2002;29:2745-52. https://doi.org/10.1118/ 1.1517611.

[14] Martins JC, Saxena R, Neppl S, Alhazmi A, Reiner M, Veloza S, et al. Optimization of Phase Space files from clinical linear accelerators. Phys Med 2019;64:54-68. https://doi.org/10.1016/j.ejmp.2019.06.007. 